\title{
Reproducibility and clinical utility of tendon palpation to detect patellar tendinopathy in young basketball players
}

\author{
J L Cook, K M Khan, Z S Kiss, C R Purdam, L Griffiths, for the Victorian Institute of \\ Sport tendon study group
}

School of

Physiotherapy, Latrobe University, Bundoora, Australia 3083

J L Cook

Department of Family Medicine (Sports

Medicine) and School of Human Kinetics, University of British Columbia, Vancouver, Canada

K M Khan

East Melbourne Radiology, East Melbourne, Australia Z S Kiss

Australian Institute of Sport, Canberra, Australia

C R Purdam

Griffith University, Southport, Australia L Griffiths

Correspondence to:

J L Cook

cook.evans@bigpond.com

Accepted 3 August 2000

\begin{abstract}
Background-Palpation is an important clinical test for jumper's knee.

Objectives-To $(a)$ test the reproducibility of palpation tenderness, $(b)$ evaluate the sensitivity and specificity of palpation in subjects with clinical symptoms of jumper's knee, and (c) determine whether tenderness to palpation may serve as a useful screening test for patellar tendinopathy. The yardstick for diagnosis of patellar tendinopathy was ultrasonographic abnormality.

Methods-In 326 junior symptomatic and asymptomatic athletes' tendons, palpation was performed by a single examiner before ultrasonographic examination by a certified ultrasound radiologist. In 58 tendons, palpation was performed twice to test reliability. Tenderness to palpation was scored on a scale from 0 to 3 where 0 represented no pain, and 1, 2, and 3 represented mild, moderate, and severe tenderness respectively.
\end{abstract}

Results-Patellar tendon palpation was a reliable examination for a single examiner (Pearson $r=0.82$ ). In symptomatic tendons, the positive predictive value of palpation was $68 \%$. As a screening examination in asymptomatic subjects, the positive predictive value of tendon palpation was $36-38 \%$. Moderate and severe palpation tenderness were better predictors of ultrasonographic tendon pathology than absent or mild tenderness $(p<0.001)$. Tender and symptomatic tendons were more likely to have ultrasound abnormality than tenderness alone $(p<0.01)$.

Conclusions-In this age group, palpation is a reliable test but it is not cost effective in detecting patellar tendinopathy in a preparticipation examination. In symptomatic tendons, palpation is a moderately sensitive but not specific test. Mild tenderness in the patellar tendons in asymptomatic jumping athletes should be considered normal.

(BrF Sports Med 2001;35:65-69)

Keywords: patellar tendon; ultrasound; palpation; reliability; athletes

Jumper's knee is a clinical condition of pain and subsequent dysfunction in the patellar tendon and the bony attachments. In the literature, this term is used interchangeably with terms that allude to the histopathology of the condition-for example, patellar tendinitis, tendinosis, and tendinopathy. In this study, the term patellar tendinopathy refers specifically to the clinical picture of jumper's knee combined with characteristic imaging changes.

In clinical practice, jumper's knee is diagnosed by history, knee examination, and palpation of the tendon and its attachments. The patellar tendon is easily palpated, as it lies immediately beneath the skin and has no substantial peritendon. The diagnosis is confirmed with imaging, commonly ultrasonography (US) and magnetic resonance imaging (MRI). Patellar tendon pain, tenderness, and imaging changes are most commonly found at the junction of the inferior pole of the patellar and the tendon attachment. ${ }^{12}$

It has been argued that palpation is the "hallmark of patellar tendinitis". ${ }^{-5}$ Duri et $a l^{6}$ found palpation to be the only sign positive in all 27 subjects in a comprehensive biomechanical and clinical examination. This cannot be used to suggest that palpation is $100 \%$ specific for jumper's knee as these subjects were diagnosed as having the condition clinically, and there was no imaging or histopathological yardstick. Thus tenderness on palpation is an important diagnostic test but there has, to our knowledge, been no critical evaluation of either its reproducibility or validity compared with any yardstick.

Although it is not feasible to use histopathology as a yardstick in clinical studies, abnormal US and MRI appearances correlated with the pathology of tendinosis in every case where patients underwent surgery. ${ }^{78}$ Thus we contend that tendon US can serve as a legitimate surrogate measure ("criterion measure") for the presence of abnormal tendon morphology. ${ }^{9-11}$ Abnormal imaging morphology, however, does not necessarily mean that symptoms must be present. ${ }^{12}$

The aims of the present study were threefold: firstly, to test the reproducibility of palpation tenderness of the patellar tendon in an at risk population of athletes; secondly, to evaluate the sensitivity and specificity of palpation as an examination technique in subjects with symptoms of jumper's knee, using US as the criterion measure; thirdly, to determine whether palpation tenderness may serve as a useful screening test for US lesions consistent with patellar tendinopathy in an asymptomatic population of at risk athletes - that is, preparticipation examination. 
Materials and methods

SUBJECTS AND CLINICAL DATA

One investigator (JLC) palpated the patellar tendons of 163 ( 80 male, 83 female) junior athletes immediately before a US radiologist (ZSK) examined them with US. All subjects completed a questionnaire that included the Nirschl scale of knee pain and function, and indicated on knee diagrams the location of symptoms. The Nirschl scale consists of a score of $0-7$ where 0 represents no symptoms, 1-4 represent increasing symptoms with activity, and 5-7 denote symptoms with daily activities and at rest. ${ }^{13}$

Current symptoms were considered present if the athlete had a Nirschl score of 1 or more and the area of pain marked on the knee diagram was directly over the proximal part of the patellar tendon. If the subject recorded the site of pain anywhere else on the knee diagram, he/she was excluded from the current symptoms group.

Palpation of the patellar tendon attachment to the patella was performed by tilting the inferior pole of the patella anteriorly. ${ }^{3}$ Central, medial, and lateral aspects of the attachment were examined. Subjects were asked to grade palpation tenderness on a scale from 0 to 3, where $0=$ no tenderness, $1=$ mild tenderness, $2=$ moderate tenderness, and $3=$ severe tenderness.

Palpation was limited to the proximal third of the tendon and the attachment to the patella. Clinically, the inferior pole of the patella and the proximal tendon are referred to most commonly in the literature on knee examination. $^{3} 1415$

In basketball players, ${ }^{12}$ and in most jumping athletes, patellar tendon pain presents mainly at the inferior pole of the patella. The relative proportions are quantified as $65 \%$ at that site, $25 \%$ at the quadriceps tendon, and $10 \%$ at the tibial insertion. ${ }^{16}$

Therefore we investigated the clinical utility of palpation as an examination technique for pain and pathology at the inferior pole of the patella.

US ASSESSMENT

In all subjects, a board certified US radiologist obtained US images in both the longitudinal and axial planes using a high resolution linear array $10 \mathrm{MHZ}$ ultrasound transducer (Acoustic Imaging Dornier; ATL Melbourne, Vic, Australia). Blinded to the subject's clinical status, the radiologist recorded the presence or absence of a US lesion using routine clinical criteria. Subjects with abnormal imaging other than at the inferior pole of the patella (three mid tendon imaging abnormalities) or with bony irregularity at the inferior pole (two tendons) were excluded from analysis. Subjects with evidence of Osgood-Schlatter disease were included in the study. The quadriceps tendon was not examined clinically or ultrasonographically.

RELIABILITY STUDY

Four hours after the tendon palpation described above, the same examiner (JLC)

\begin{tabular}{|c|c|c|c|c|}
\hline \multirow{2}{*}{$\begin{array}{l}\text { Second palpation } \\
\text { score }(n=58)\end{array}$} & \multicolumn{4}{|c|}{ First palpation score $(n=58)$} \\
\hline & 0 & 1 & 2 & 3 \\
\hline 0 & 9 & 4 & 0 & 0 \\
\hline 1 & 0 & 17 & 4 & 0 \\
\hline 2 & 0 & 1 & 12 & 2 \\
\hline 3 & 0 & 1 & 4 & 4 \\
\hline
\end{tabular}

re-palpated 29 randomly selected subjects (58 tendons). Subjects were inactive between tests. The US examination took place after palpation and the examiner was blinded to the results of palpation in the first test.

\section{STATISTICAL METHODS}

Data were analysed using Excel 5.0 (Microsoft Corporation, Redmond, WA, USA). Patient characteristics and mean dimensions of hypoechoic regions were calculated using descriptive statistics. A $2 \times 2$ contingency table was used to test palpation in normal and abnormal tendons. Student's $t$ test was used to test significance in symptomatic and asymptomatic tendons. Significance levels were set at $\mathrm{p}<0.05$.

\section{Results}

DESCRIPTIVE CHARACTERISTICS

The subjects had a mean (SD) height of 185.1

(11.3) cm, weight of $75.6(11.8) \mathrm{kg}$ and age of 197.2 (12.2) months. The age range was 14.218.7 years.

PALPATION RELIABILITY

Table 1 shows the reproducibility of the test-retest palpation. Overall reproducibility of palpation was 0.82 (Pearson correlation coefficient). Forty one of 58 test-retest results were identical. In 16 of the 17 (94\%) remaining cases palpation scores differed by one point only. Results that follow exclude the second patellar tendon palpation.

CLINICAL UTILITY OF PALPATION IN ATHLETES WITH THE CLINICAL SYNDROME OF JUMPER'S KNEE

There were current symptoms in 27 tendons. Of these, 16 contained a US lesion and $11 \mathrm{did}$ not. In symptomatic subjects, the sensitivity of palpation was $68 \%$ and specificity was $9 \%$. The positive and negative predictive values of palpation in this group were $52 \%$ and $17 \%$ respectively (table 2 ).

We compared the incidence of US abnormality in tender tendons (Nirschl score 1-3) with and without symptoms (table 3). Tendons with both symptoms and tenderness were significantly more likely to have US abnormality than those with palpation tenderness and no symptoms $(\mathrm{p}<0.01)$. If we excluded mild

Table 2 Symptomatic tendons: palpation vs ultrasonographic (US) appearance

\begin{tabular}{lll}
\hline & US lesion & US normal \\
\hline Tender & 11 & 10 \\
Not tender & 5 & 1 \\
\hline
\end{tabular}

$\chi^{2}=1.85$. Not significant. 
Table 3 Ultrasonography (US) results in tender tendons (1-3) with and without symptoms

\begin{tabular}{lll}
\hline & US lesion & US normal \\
\hline Symptomatic & 11 & 10 \\
Asymptomatic & 35 & 126 \\
\hline$\chi^{2}=9.2 . \mathrm{p}<0.01$. & &
\end{tabular}

Table 4 Ultrasonography (US) results in tender tendons (2-3) with and without symptoms

\begin{tabular}{lll}
\hline & US lesion & US normal \\
\hline Symptomatic & 11 & 10 \\
Asymptomatic & 23 & 39 \\
\hline$\chi^{2}=1.5 . \mathrm{p}>0.05$. & &
\end{tabular}

Table 5 Palpation scores in asymptomatic athletes with and without ultrasonographic (US) lesions in the patellar tendon

\begin{tabular}{lllll}
\hline \multicolumn{5}{l}{ Palpation scores } \\
& 1 & 2 & 3 & 4 \\
& Not tender & Mild & Moderate & Severe \\
\hline US normal $(\mathrm{n}=236)$ & 110 & 87 & 30 & 9 \\
US lesion $(\mathrm{n}=63)$ & 28 & 12 & 18 & 5 \\
Predictive value & $80 \%{ }^{\star}$ & $12 \% \dagger$ & $38 \% \dagger$ & $36 \% \dagger$ \\
\hline
\end{tabular}

*This represents a negative predictive value-that is, the finding of no tenderness on palpation means that there is an $80 \%$ likelihood that there is no US lesion.

†This represents a positive predictive value-that is, the likelihood that this degree of tenderness is associated with a US lesion.

Table 6 Summary of palpation results and ultrasonographic (US) findings in basketball players with asymptomatic patellar tendons

\begin{tabular}{lll}
\hline & US lesion & US normal \\
\hline Tender $(1-3)$ & 35 & 126 \\
Not tender $(0)$ & 28 & 110 \\
\hline
\end{tabular}

US results in tendons that were not tender (0) and in those that had some tenderness (grades $1-3) \cdot \chi^{2}=0.09$. Not significant.

tenderness (Nirschl score 1) from the tender group, then tenderness and symptoms was not associated with US abnormality $(p>0.05)$ (table 4).

CLINICAL UTILITY OF PALPATION AS A SCREENING TEST: PALPATION AND THE PREPARTICIPATION EXAMINATION

Of the 299 asymptomatic tendons, 63 contained a US lesion and 236 did not (table 5). We calculated sensitivity and specificity of palpation scores in two clinically relevant ways. Firstly, we classified only the palpation finding of no pain as "not tender" (0), and grouped all the tenderness categories (1,2 and 3 ) as "tender" (table 6). With this definition of "tenderness", palpation had a sensitivity of $56 \%$ and specificity of $47 \%$. The positive predictive value was $22 \%$.

Secondly, we combined subject tendons with no tenderness (0) and mild tenderness (1) and compared them with subject tendons that had either moderate (2) or severe (3) tenderness (table 7 ). When grouped in this way, moderate and severe tenderness had a sensitivity of $37 \%$ and specificity of $83 \%$. Positive predictive value was $37 \% \cdot \chi^{2}$ analysis showed that moderate and severe tenderness was a significantly better predictor of US abnormality than was zero or mild tenderness $(\mathrm{p}<0.001)$.
Table 7 Ultrasonographic (US) results in tendons that were not tender or mildly tender (0 or 1) and those that were moderately or severely tender (tenderness grades 2, 3)

\begin{tabular}{lll}
\hline & US lesion & US normal \\
\hline Tender $(2-3)$ & 23 & 39 \\
Not tender $(0-1)$ & 40 & 197 \\
\hline$\chi^{2}=12.08 . \mathrm{p}<0.001$. & &
\end{tabular}

$\chi^{2}=12.08 . \mathrm{p}<0.001$

\section{Discussion}

Tenderness on palpation is a key component of the clinical diagnosis of patellar tendinopathy. The present study is, to our knowledge, the first to examine the reliability of this important clinical sign or its clinical utility in the diagnosis of patellar tendinopathy in patients with symptomatic jumper's knee. Further, we tested the utility of palpation as a test in the preparticipation examination to detect underlying US tendon lesions.

\section{ASYMPTOMATIC LESIONS}

Asymptomatic imaging abnormalities have been shown in the tendons of athletes playing volleyball, basketball, and soccer and of track and field athletes. ${ }^{212}{ }^{17-19}$ Leadbetter $^{20}$ postulated that degenerative tendon pathology could remain asymptomatic for a period when he defined tendinopathy as "a focal intratendinous degeneration that may or may not be symptomatic". Support for the concept of asymptomatic tendinopathy is found in the study of 891 spontaneous tendon ruptures. ${ }^{21}$ Of these, 97\% had histopathological evidence of degeneration (tendinosis). However, only $34 \%$ of these tendons were symptomatic before rupture.

US is used extensively to image tendons, and clinically, abnormal US appearance is considered to represent abnormal tendon tissue. ${ }^{22}$ US characteristically reveals swelling of the tendon and loss of fascicular continuity, and this corresponds histologically to collagen degeneration with increased ground substance and vascularity. ${ }^{23}$ The imaging appearance of asymptomatic tendinopathy is indistinguishable from symptomatic tendinopathy. ${ }^{1}$ There are, as yet, no studies that show the histopathology of asymptomatic imaging abnormalities.

\section{DO TENDONS WITH ASYMPTOMATIC IMAGING} ABNORMALITIES BECOME SYMPTOMATIC? A longitudinal study of basketball players in the same age group as this study showed that symptoms at baseline conferred 4.2 times greater risk of developing symptoms than normal tendon at baseline. ${ }^{24}$ Similar studies in adult female basketball players also showed that a hypoechoic tendon at baseline conferred an increased risk of symptoms at a 17 month follow up. ${ }^{25}$

\section{IS PALPATION A RELIABLE EXAMINATION?}

In the hands of a single examiner, palpation can reliably grade tenderness on a $0-3$ scale. Further investigation is needed to examine intertester reliability. 
IS PALPATION AN EFFECTIVE CLINICAL EXAMINATION TOOL IN PATIENTS WHO HAVE SYMPTOMS OF JUMPER'S KNEE?

In this group of elite junior athletes, if US evidence of tendon lesions was used as a criterion, palpation was a moderately sensitive but not specific test for patellar tendinopathy. Symptomatic tendons with palpation tenderness were more likely to have US abnormality than asymptomatic tendons with palpation tenderness. However, if tendons with mild tenderness were excluded, then tenderness in association with symptoms was not an indication of US status.

IS PALPATION A USEFUL COMPONENT OF THE PREPARTICIPATION EXAMINATION TO DETECT ASYMPTOMATIC US LESIONS IN THE PATELLAR TENDON?

Tenderness on palpation is not a useful predictor of a US lesion in the patellar tendons of high risk, but asymptomatic, subjects. This is the population that undergoes the preparticipation examination. The positive predictive value of tenderness was low for both the mild to severe classification (tenderness grades 1, 2, and 3 ) and the moderate and severe classification (tenderness grades 2 and 3 only). $\chi^{2}$ was significant when moderate and severe palpation tenderness was required before palpation was considered positive. Our data indicate that elite heavily training young athletes may have mild tendon soreness without US tendon pathology. Although no tenderness on palpation was strongly associated with normal tendons, the purpose of a preparticipation examination is to detect modifiable pathology.

SINDING-LARSEN-JOHANSSON DISEASE

Although Sinding-Larsen-Johansson (SLJ) disease in girls of this age cannot be totally discounted, it is generally considered to occur at an earlier age than that of the subjects in this study (10-11 years). Almost all of the boys in this study were outside the age group for SLJ in males (13-14 years). ${ }^{26}$ US imaging of this condition can disclose abnormal morphology such as bony irregularity and tendon swelling. ${ }^{27}$

\section{CLINICAL IMPLICATIONS}

(1) In subjects of this age group, palpation is a reliable examination technique. Thus further research into its clinical utility in various populations is warranted.

(2) As a diagnostic test in subjects presenting with symptoms consistent with jumper's knee, the sensitivity and specificity of tendon palpation were $68 \%$ and $9 \%$ respectively, suggesting that the test is only somewhat useful in the diagnostic process. We found that moderate and severe tenderness (positive predictive value $36 \%$ and $38 \%$ respectively) were better predictors of patellar tendinopathy than was zero or mild tenderness (positive predictive value $<20 \%$ ). This implies that clinicians should not attach undue significance to mild tenderness at the patellar tendon attachment site.

(3) As a part of the preparticipation examination in asymptomatic, but high risk subjects, moderate and severe palpation tenderness detected $37 \%$ of US lesions-that is, there is a false positive rate of $63 \%$. Thus, if bilateral patellar US scans are costed at f50, screening 100 tendons would cost $£ 2500$. In a population such as reported in this study, and in others, the prevalence of tendons with asymptomatic US lesions is about $20 \% .^{12}$ A positive predictive value of $40 \%$ would lead to detection of only eight of the 20 abnormal tendons, at a cost of over $£ 300$ per tendon. Furthermore, 12 US abnormal tendons would remain undetected because palpation tenderness was absent. Given that the clinical significance of asymptomatic US lesions remains unclear, ${ }^{212}$ our data provide no indication that patellar tendon tenderness at preparticipation examination should be followed by US.

Investigators in the Victorian Institute of Sport tendon study group are: Departments of Medicine and Radiology, Royal Melbourne Hospital; Physiotherapy School, LaTrobe University; Australian Institute of Sport; Victorian Institute of Sport; East Melbourne Radiology; J Bartlett, F Bonar, B Coleman, J Cook, K Crichton, M Fehrmann, M Grant, L Griffiths, P Harcourt, D Kellaway, K Khan, Z S Kiss, C Purdam, B Tress, P Visentini, J Wark, D Young. The following institutions are represented: Department of Medicine, University of Melbourne, resented: Department of Medicine, University of Melbourne, Royal Melbourne Hospital; Department of Radiology, Univertute of Sport; Victorian Institute of Sport.

1 Cook J, Coleman B, Khan K, et al. Patellar tendinosis in junior basketball players: A controlled clinical and ultrasonographic study of 268 tendons in players aged 14-18 years. Scand $\mathcal{f}$ Med Sci Sports 2000;10:216-22.

2 Cook JL, Khan KM, Harcourt PR, et al. Patellar tendon ultrasonography in asymptomatic active athletes reveals hypoechoic regions: a study of 320 tendons. Clin 7 Sports hypoechoic region:
Med 1998;8:73-7.

Med 1998;8:73-7.
3 Colosimo AJ, Bassett FH. Jumper's knee: diagnosis and treatment. Orthop Rev 1990;29:139-49.

4 Fritschy D. Jumper's knee. Operative Techniques in Sports Medicine 1997;5:150-2.

5 Roels J, Martens M, Mulier JC, et al. Patellar tendinitis (jumper's knee). Am f Sports Med 1978;6:362-8

6 Duri ZAA, Aichroth PM. Patellar tendonitis: clinical and literature review. Knee Surgery Sports Traumatology Arthroscopy 1995;3:95-100.

7 Khan KM, Bonar F, Desmond PM, et al. Patellar tendinosis (jumper's knee): findings at histopathologic examination, US and MR imaging. Radiology 1996;200:821-7.

8 Green JS, Morgan B, Lauder I, et al. Correlation of magnetic Green JS, Morgan B, Lauder I, et al. Correlation of magnetic
resonance imaging and histology in patellar tendinitis. resonance imaging and histology in
Sports Exercise and Injury 1997;3:80-4.

9 Davies SG, Baudovin CJ, King JB, et al. Ultrasound, Davies SG, Baudovin CJ, King JB, et al. Ultrasound,
computed tomography and magnetic resonance imaging in patellar tendinitis. Clin Radiol 1991;43:52-6.

10 Laine HR, Harjula ALJ, Peltokallio P. Ultrasound in the evaluation of the knee and patellar regions. F Ultrasound Med 1987;6:33-6.

11 Mourad K, King J, Guggiana P. Computed tomography and ultrasound imaging of jumper's knee - patellar tendinitis. Clin Radiol 1988;39:162-5.

12 Lian O, Holen KJ, Engebrestson L, et al. Relationship between symptoms of jumper's knee and the ultrasound characteristics of the patellar tendon among high level male volleyball players. Scand 7 Med Sci Sports 19966:291-6.

13 Nirschl RP. Elbow tendinosis/tennis elbow. Clin Sports Med 1992;11:851-70

14 Puddu G, Franco V, Selvanetti A., et al. Jumper's knee and other forms of tendinitis about the knee. In: The Hughston clinic sports medicine book. Baltimore: Williams and Wilkins, clinic sports medici

15 Zini R, Coari GC. Arthroscopic management of patellar tendinitis. Fournal of Sports Traumatology and Related

16 Perugia L, Ippolito E, Postacchini F. Patologia e clinical delle lesioni tendinea da sport. Medicina dello Sport 1977;30:85-116.

17 Gibbon WW, Cooper JR, Radcliffe JS. Sonographic ncidence of tendon microtears in athletes with chronic Achilles tendinosis. Br F Sports Med 1999;33:129-30.

18 Rollandi GA, Bertolotto M, Perrone R, et al. MRI of normal Achilles tendon. European Radiology 1995;5596-8.

19 Soila K, Karjalainen PT, Aronen H, et al. High-resolution MR imaging of the asymptomatic Achilles tendon: new observations. American fournal of Roentgenology 1999;173: 323-8.

20 Leadbetter W. Cell matrix response in tendon injury. Clin Sports Med 1992;11:533-78.

21 Kannus P, Jozsa L. Histopathological changes preceding spontaneous rupture of a tendon. $\mathcal{F}$ Bone foint Surg [Am] 1991;73:1507-25. 
22 Lisle DA. Musculoskeletal ultrasound in sports medicine Sports Link 1999 Sep:9-14.

23 Khan KM, Bonar SF, Cook JL, et al. Histopathology of common overuse tendon conditions: update and implications for clinical management. Sports Med 1999;6: 393-408.

24 Cook J, Kiss Z, Khan K, et al. Patellar tendon ultrasound in elite junior basketnall players: a 12 month longitudina study in 52 tendons. F Ultrasound Med 2000;19:473-9.

Take home message

This study indicates that palpation of the patellar tendon may not offer the clinician diagnostic certainty of tendon pathology as shown on US examination. An accurate history and other examination techniques should be used to confirm the diagnosis in symptomatic athletes. Palpation tenderness does not indicate US status in asymptomatic athletes and should not be used to screen athletes for potential problems in this tendon.
25 Khan KM, Cook JL, Kiss ZS, et al. Patellar tendon ultrasonography and jumper's knee in elite female basket199-206.

26 Micheli LJ. The traction apophysites. Clin $\mathcal{F}$ Sports Med 1987;6:389-404.

27 Barbuti D, Bergami G, Testa F. Ultrasonographic aspects of Sinding-Larsen-Johansson disease. Paediatric Medicine 1995;17:61-3. 